\title{
UN MODELO PRAGMÁTICO DE LA COMUNICACIÓN ESCRITA EN EL AULA DE CIENCIAS
}

\author{
SÁNCHEZ GÓMEZ, PEDRO J. \\ Departamento de Didáctica de las Ciencias Experimentales \\ Facultad de Educación. Universidad Complutense de Madrid \\ Rector Royo-Villanova, s/n. 28040 Madrid \\ pedros@eucmos.sim.ucm.es
}

Resumen. En este artículo presentamos un modelo pragmático aproximado de la comunicación escrita en el aula de ciencias. Nos hemos basado eminentemente en las ideas de Umberto Eco sobre la interpretación de textos. Hemos denominado a este modelo aproximación interpretativa (ApIn). El modelo ApIn nos lleva a una nueva visión de la realidad de aula, que trae consigo una reinterpretación de muchos aspectos didácticos y, muy en especial, de la evaluación del aprendizaje. A su vez, el enfoque pragmático permite estudiar de una manera original el problema de la relación entre lenguaje científico y lenguaje ordinario en la enseñanza de las ciencias.

Palabras clave. Didáctica de las ciencias, filosofía del lenguaje, evaluación didáctica, escritura científica.

Summary. In this paper, we present an approximated pragmatic model of written communication in the science classroom. It is mainly based on Umberto Eco's ideas on text interpretation. We have called this model Interpretative Approximation (ApIn, for the Spanish, aproximación interpretativa). The ApIn model brings about a re-interpretation of many didactic features and, in particular, of didactic assessment. Besides, this pragmatic approach allows a fresh view on the problem of the relationship between scientific and ordinary language in the framework of science teaching.

Keywords. Science teaching, philosophy of language, didactic assessment, scientific writing.

\section{INTRODUCCIÓN}

El lenguaje es la piedra angular sobre la que descansa todo el proceso de enseñanza-aprendizaje. No se trata tan sólo de que la adquisición de una serie de destrezas lingüísticas sea un fin fundamental de toda la enseñanza, sino también de que todo aprendizaje pasa necesariamente por una comunicación y, por lo tanto, de una interacción lingüística. Pero el lenguaje es mucho más que un simple medio para la interacción de aula. Como dice Rowell (1997), centrándose en el campo concreto de la didáctica de las ciencias, «en las escuelas, como en otros entornos, es difícil separar el aprendizaje del lenguaje, el aprendizaje a través del lenguaje y el aprendizaje sobre el lenguaje [...] El lenguaje de las lecciones de ciencia es el medio en el cual la ciencia como una actividad humana (y su correspondiente cuerpo de conocimientos) es integrado por muchos de los estudiantes». Aprender ciencias supone o implica o, incluso, consiste en aprender el lenguaje de la ciencia (Halliday y Martin, 1993, p. 84). 
El estudio de los aspectos discursivos y lingüísticos está cobrando una importancia cada vez mayor dentro de la investigación en didáctica de las ciencias (Izquierdo y Sanmartí 2000, pp. 181-193; Sanmartí et al., 1999). Cuando hablamos del lenguaje en el aula, estamos en realidad hablando de varias actividades distintas, con matices e implicaciones diferentes: hablar, leer y escribir. Como destaca Rowell (1997, p. 20), remitiéndose a Russell (1991) y a Sperling (1996), de estas tres actividades, es la escritura la que menos atención ha recibido tradicionalmente en el marco de la investigación en didáctica de las ciencias. Sin embargo, en los últimos años, esta situación está comenzando a cambiar -podemos observar, por ejemplo, Jorba y otros (1998); y a su vez, como revisión de los trabajos recientes en este sentido, junto con el artículo de Rowell (1997) que hemos venido citando, también podemos citar a Rivard (1994)-. Este interés creciente, sin duda alguna, está motivado en parte por el impulso que han cobrado en los últimos años los estudios sobre el discurso de la ciencia y, más en concreto, sobre la escritura científica (Halliday y Martín, 1993; Solomon, 1994; Sutton,1992; 1996a; 1996b). Dentro de esta tendencia, en los últimos años se está consolidando una línea de pensamiento que pone en tela de juicio la visión «tradicional» de los textos científicos, según la cual, al menos idealmente, la escritura científica no es más que un vehículo objetivo y «aséptico», sin ninguna carga subjetiva. Frente a esta postura, estos nuevos estudios inciden en los aspectos personales de los textos científicos, y algunos autores llegan a proponer una relectura de los mismos desde la perspectiva de los estudios literarios (Locke, 1992). Obviamente, no se trataría de asimilar los artículos científicos a las obras literarias, sino más bien emplear la metodología y el corpus teórico de los estudios literarios en el análisis de la obra científica.

Otro de los orígenes del interés por la escritura en la enseñanza-aprendizaje de las ciencias es el papel central que en los últimos años los aspectos discursivos están tomando en la psicología cognitiva (Scott, 1998). El hito más destacable de este nuevo rumbo es, seguramente, el libro Acts of Meaning, de Bruner (1990). En esta obra, los aspectos socioculturales, como mediadores esenciales en la formación de significados, son tomados como variables centrales para el estudio psicológico. Como destaca Scott (1998), las raíces de esta nueva psicología discursiva (o cultural) tienen precedentes tan ilustres como el Wittgenstein de las Investigaciones filosóficas, el interaccionismo simbólico de Mead y los trabajos de Vygotsky, Luria y Leontiev. En el campo de los estudios didácticos, esta nueva orientación se traduce en un interés creciente en los mecanismos de construcción de significados en la interacción social de aula, y ha dado lugar a una serie de enfoques teóricos que se suelen englobar bajo el epígrafe de constructivismo social o constructivismo contextual (Cobern, 1993). Desde esta perspectiva, la producción de materiales escritos por parte de los alumnos, aparte de un método «metacognitivo» (y, por lo tanto, personal) de aprendizaje (Applebee, 1984), es vista como una práctica social dentro de una comunidad determinada (Lemcke, 1995). Los escritos de los alumnos tienen, por lo tanto, un interés intrínseco, que va más allá de su valor como instrumentos de aprendizaje, como constituyentes fundamentales de la dinámica social de aula.
Sin duda alguna, todas estas nuevas orientaciones teóricas han contribuido a profundizar en los últimos años en muchos de los aspectos de la enseñanza-aprendizaje de las ciencias, pero lo cierto es que la escritura ha jugado, desde siempre, un papel central tanto en la práctica docente como en la investigación didáctica. En primer lugar, hay un acuerdo tradicional en el papel de la escritura como habilidad instrumental básica que debe ser adquirida, como objetivo fundamental, en la enseñanza obligatoria (habilidad que debe ser desarrollada en los niveles superiores de la educación): la alfabetización es, tal vez, el objetivo prioritario de la educación, por mucho que hoy exijamos a las escuelas mucho más que una «mera» alfabetización. En segundo lugar, los materiales escritos han sido siempre, y aún son hoy, el medio fundamental empleado en la evaluación del proceso de enseñanza-aprendizaje. «Los exámenes», por muy controvertidos que puedan resultar, son una de las características definitorias de la escuela. A su vez, no hay que olvidar que una parte muy importante de la investigación didáctica, sobre todo la centrada en la exploración del pensamiento del alumno (una línea especialmente relevante dentro de la didáctica de las ciencias), está basada, en una proporción muy alta, en el estudio de materiales escritos. Por último, e íntimamente relacionado con lo anterior, hay que destacar que una parte muy importante de la investigación didáctica, desarrollada desde y para la enseñanza de las ciencias, se ha basado en el desarrollo y la evaluación de métodos gráficos para la producción y la representación de expresiones lingüísticas. Entre estos métodos, cabe destacar el de los «mapas conceptuales» (Novak, 1984, 1990; Pankratius, 1990), así como el de las «redes semánticas» (Galagovsky, 1993, 1994, 1996).

Los textos generados en el aula, en definitiva, constituyen un material de primer interés en la didáctica de las ciencias. Este interés por la palabra escrita no es, obviamente, patrimonio exclusivo de la didáctica o de la filosofía de la ciencia. De hecho, los estudios textuales constituyen uno de los campos de trabajo más activos del pensamiento actual. Una de las líneas más interesantes en este sentido es la que podríamos denominar semiótica textual (Eco, 1981). Este enfoque pretende analizar los textos dentro del marco de la teoría semiótica, esto es, como actos de comunicación formados por signos, en este caso de naturaleza lingüística, integrados en códigos. El enfoque semiótico, de hecho, rebasa ampliamente el marco de los estudios textuales. La semiótica es, tal vez, el modelo para el estudio de la cultura más coherente y comprehensivo (Eco, 1977). En esta línea, algunos autores han adoptado un enfoque semiótico para el estudio de problemas didácticos, especialmente dentro del área de la didáctica de las matemáticas (Godino y Batanero, 1994, 1997). En lo referente a la didáctica de las ciencias, hay que destacar el notable artículo de Groisman (1991), en el que se estudian, desde la perspectiva de la semiótica, diferentes aspectos de la cultura de aula. A su vez, podemos señalar las últimas investigaciones de Weinninger sobre la semiótica del lenguaje de la química (Weinninger, 1998), que, aunque no están orientadas primariamente a la didáctica, tienen implicaciones inmediatas en la enseñanza.

En este artículo presentamos un modelo de la comunicación didáctica que tiene lugar en torno a la escritura de 
textos en el aula de ciencias. Nos basaremos de manera principal en las ideas sobre la interpretación de textos publicadas por Eco $(1981,1998)$. Hemos organizado el artículo tal como explicamos a continuación. En la sección 2, presentamos un resumen de los aspectos más relevantes de la teoría de la interpretación textual de Umberto Eco. Basándonos en estas ideas, en la sección 3 presentamos nuestro modelo aproximado de comunicación escrita en el aula de ciencias, al que denominaremos Aproximación interpretativa (ApIn). En esta sección analizamos en profundidad las bases teóricas del modelo, para estudiar sus implicaciones didácticas en la sección 4. Hemos reservado una sección entera, la quinta, a la visión de la evaluación didáctica a la que da lugar el modelo ApIn. Por último, en la sección 6 desarrollamos lo que, a nuestro juicio, es uno de los puntos más importantes de nuestra propuesta: la integración de un enfoque semántico y otro pragmático en el marco de la enseñanza de las ciencias.

\section{LA INTERPRETACIÓN DE UN TEXTO DE ACUERDO CON UMBERTO ECO}

Para estudiar el papel de los textos de los alumnos es conveniente encuadrar el tema dentro del contexto más general de la interpretación de un texto. El problema de la interpretación es una de las cuestiones abiertas del pensamiento contemporáneo y, lógicamente, no es el objeto de este artículo entrar en los detalles de este tema (un tema, además, particularmente complejo). Baste a nuestros propósitos destacar los aspectos que, a nuestro juicio, resultan más relevantes de cara al análisis del contenido de los textos de los alumnos. En la argumentación que sigue, hemos tomado como guía los espléndidos estudios al respecto que Umberto Eco ha publicado (1981, 1998).

Eco concibe la interpretación como una búsqueda de una respuesta a la pregunta ¿Cómo funciona un texto? Para contestar esta pregunta, es necesario, en primer lugar, determinar el papel que juegan las piezas de las que consta la máquina textual (autor, lector) y el texto en sí. Eco comienza por señalar una serie de posturas generales que los estudiosos han tomado históricamente sobre la interpretación (Eco, 1998, p. 29). Así, distingue una tricotomía entre: $a$ ) interpretación como búsqueda en el texto de lo que el autor quiere decir (búsqueda de la intentio auctoris; el texto funciona, eminentemente, por el autor que hay detrás de él); b) como búsqueda de lo que el texto dice, independientemente de las intenciones del autor, con referencia a su misma coherencia contextual y a la situación de los sistemas de significación a los que se remite (búsqueda de la intentio operis; el texto funciona por sí mismo); y, c) por último, como proceso en el que el lector, en función de sus motivaciones, su formación, etc., da sentido a un texto concreto (intentio lectoris; el lector es el que hace funcionar al texto).

Para Eco, la interpretación de un texto debe ser concebida como la elaboración de una conjetura sobre la intentio operis. Destaquemos que el término conjetura es empleado en un sentido explícitamente popperiano: la interpretación puede (y debe) ser falsada dentro del mismo texto tomado como un todo. Esto no restringe a priori la posibilidad de que se puedan dar interpretaciones múltiples, incluso infinitas, pero descarta aquéllas que no se sostienen dentro del texto en su conjunto.

Otro punto fundamental del análisis de Eco es la distinción entre lector modelo y lector empírico. Según esta idea, todo texto (y en general todo material comunicativo) construye su lector modelo, un lector hipotético capaz de «sintonizar» con el texto, de darle una interpretación adecuada. Un texto puede implicar más de un lector modelo, y puede ocurrir también que el lector (o los lectores) modelo que construye un texto no coincida con el lector empírico que, de hecho, lo esté leyendo. Un ejemplo sencillo de todo esto podría ser la frase «El gato que está dentro de la caja está vivo y muerto a la vez». Esta frase puede parecer paradójica, o incluso sin sentido, para muchos lectores, pero tiene connotaciones inmediatas para un lector versado en mecánica cuántica, y conocedor de la paradoja de Schrödinger. A su vez, un psiquiatra podría leerla como una muestra del discurso de un esquizofrénico; y también sería posible que una persona la leyera como un aforismo poético. En definitiva, esta frase implica, al menos, tres lectores modelo y, a su vez, es muy probable que el lector empírico no coincida con ninguno de los tres. Como se ve, el postulado de un lector modelo implica automáticamente una hipótesis sobre el autor del texto. Por lo tanto, de igual modo que distinguimos entre lector empírico y lector modelo, debemos diferenciar un autor empírico y un autor modelo. La interpretación, como búsqueda de una intentio operis, implica, de hecho, una integración de las tres intentiones que veíamos antes. Cualquier hipótesis sobre la intentio operis supone, aun implícitamente, una conjetura sobre el lector modelo que el texto construye y, por lo tanto, sobre el autor modelo del mismo (y sobre las intentiones de este lector y de este autor modelo).

La interpretación de un texto supone una actualización del mismo por parte del lector. Por actualización se entiende los «movimientos» que el lector efectúa para dar sentido a la manifestación lineal de significantes léxicos que constituye el texto. Un aspecto central de la teoría semiótica de la interpretación es que esta actualización no puede entenderse desde el punto de vista de una gramática de la oración (Eco, 1981, pp. 24-31). El sentido de un texto no se puede, en general, establecer a partir de un análisis de sus componentes cuando éstos se analizan como si fuesen entradas de un diccionario; esto es, cuando se tiene en cuenta únicamente su valor semántico aislado y sus propiedades sintácticas. Para interpretar un texto se requiere de un código (o códigos) organizado en forma no ya de diccionario, sino de enciclopedia, en la que cada entrada incluya información contextual e información circunstancial sobre un término dado. Información contextual se refiere a que el código registre la posibilidad de que el término aparezca asociado a otros términos del mismo sistema semiótico. Así, y volviendo al ejemplo que veíamos anteriormente, una buena enciclopedia debería contemplar que el término gato puede aparecer asociado a los términos ronroneo, arañazo o bigotes, y que en ese caso designará a un mamífero de la familia de los felinos, de nombre científico Felix Domesticus L. A su vez, debería incluir otras selecciones contextuales, como, por ejemplo, que puede 
aparecer en conexión con coche o con rueda, e incluso, otras mucho más restringidas, como que en algunas ocasiones puede asociarse a «El Foro», «Lavapiés» o «San Isidro» (entre otros); o también a caja, o a principio de superposición. Por información circunstancial se entiende la posibilidad de que un término pueda tener variaciones de sentido según las circunstancias de enunciación. Por ejemplo, el código enciclopédico debería registrar que la expresión «La caja está llena», entre otras muchas situaciones, se puede emplear en un banco, o en un casino, o en una funeraria, o en un texto de mecánica cuántica y, en cada caso, daría lugar a significados distintos.

Un texto es, como a menudo dice Eco, un «artefacto perezoso». Su actualización requiere, de la cooperación del lector para llenar de contenido su manifestación lingüística superficial. De otra manera, todo texto postula la cooperación de un lector, en principio hipotético, para su interpretación. Ya hemos puesto nombre a este lector: el lector modelo. El autor debe asumir una serie de competencias en este lector modelo hipotético para que sea capaz de dar contenido a sus expresiones y, en función de esta asunción, adoptar una estrategia textual. Estas competencias deben ser gramaticales (la más obvia es la lengua en la que se escribe el texto), pero también «enciclopédicas»: el autor y el lector han de compartir unos códigos, en el sentido enciclopédico que introducíamos en el párrafo anterior. Un lector que ignore la paradoja de Schrödinger (con sus implicaciones contextuales y circunstanciales referidas a los términos gato o caja), por muy versado que esté en mecánica cuántica, quedará, como poco, desconcertado de leer la frase que hemos puesto antes como ejemplo. De igual manera, un lector ante una determinada estrategia textual debe postular una serie de hipótesis sobre un autor modelo, en el sentido de que debe decidir de que código o códigos debe echar mano para interpretar el texto que esta leyendo (Eco, 1981, pp. 73-94): ¿la frase ha sido escrita por un físico, por un poeta o por un esquizofrénico? Cuando decíamos anteriormente que lector modelo y lector empírico no tienen por qué coincidir, nos referíamos a que, en algunos casos, las competencias demandadas por el texto pueden ser diferentes de las que posea el lector empírico. En estas situaciones, el texto puede simplemente no ser comprendido o, y esta es una posibilidad muy importante en el contexto didáctico, puede ser malinterpretado.

\section{EL MODELO DE APROXIMACIÓN, INTER- PRETATIVA DE LA COMUNICACIÓN TEX- TUAL EN EL AULA}

Ya hemos señalado en la introducción la importancia que la interpretación de textos tiene en el contexto de la didáctica de las ciencias, sobre todo en los estudios de orientación constructivista de estudio del «pensamiento» de los diferentes actores didácticos. Esta línea de investigación, con sus diversas orientaciones (Pintó et al., 1996), se ha sabido mostrar extraordinariamente productiva (Gil 1996). Por otro lado, en los últimos años, se percibe un desplazamiento en el foco de la investigación didáctica, que está pasando de centrase en aspectos eminentemente personales a hacerlo en aspectos más sociales. Es en esta tenden- cia en la que se encuadran los autores que inciden en los aspectos culturales de la enseñanza-aprendizaje de las ciencias (Lynch y Jones, 1995; Krugly-Smolska, 1995). Se podría decir que se está consolidando una línea de pensamiento en la investigación didáctica que desplaza el énfasis del alumno individual (o del docente individual) como sujeto cognoscente (Prior, 1997, pp. 63-65; Cobern, 1993, pp. 52-55) y al aula como grupo generador de una cultura. El interés de las ideas de Eco dentro de esta última perspectiva es evidente, pero, de hecho, también se extiende a los estudios constructivistas centrados en la búsqueda del pensamiento del alumno. Nos referimos a que, en este caso, la interpretación del texto sigue siendo (o, al menos, debería seguir siendo) un requisito fundamental de la investigación. En esta sección presentamos una aplicación del modelo interpretativo de Eco a los textos producidos en el aula de ciencias. De una manera sintética, pretendemos intentar responder a la pregunta «¿Cómo funcionan los textos de alumnos y docentes en el contexto del aula de ciencias?».

Cuando empleamos el verbo funcionar, estamos destacando un aspecto crucial de nuestro enfoque: pretendemos modelizar tanto de qué nos informan los textos generados en el aula como qué se pretende hacer con ellos y cómo. En definitiva, nos encuadramos dentro de la visión pragmática del estudio del significado: el lenguaje no es sólo un medio para transmitir información, sino que es eminente y primariamente un instrumento para hacer cosas. Al emplear el lenguaje no sólo describimos el mundo, sino que actuamos sobre él. Cuando alguien emplea el lenguaje, debemos plantearnos no tanto si lo que dice es o no es verdad, sino si los objetivos de su acto de habla se cumplen o no. En la sección 5 profundizaremos más en este aspecto.

Eco, cuando introduce sus reflexiones sobre la interpretación de textos (Eco, 1998), no pretende desarrollar una teoría de la psicología de la comprensión del texto escrito. Se trata más bien de marcar unas pautas para una interpretación textual científica, en el sentido de contrastable y reproducible, basada en sus estudios sobre la pragmática del lenguaje escrito (Eco, 1981). Los textos pueden ser leídos de maneras diversas, siendo la interpretación «como hipótesis falsable sobre la intentio operis» tan sólo una de ellas. De hecho, el mismo Eco, en diferentes lugares de su obra (Eco, 1981, pp. 82-87, 1998, pp. 117-120), estudia otro enfoque posible del texto: el uso, como contrapuesto a la interpretación. Un texto es usado cuando es visto como un medio, si se quiere el único, para llegar a conclusiones ajenas a él mismo. Un ejemplo cotidiano de uso es la lectura de una poesía con el fin de alcanzar un goce estético. En este caso, el lector no intenta determinar cómo funciona la poesía (no la interpreta), sino que la utiliza para procurarse una satisfacción subjetiva. Fuera de este ejemplo hasta cierto punto extremo, cabría plantearse si la forma en la que los distintos agentes didácticos (alumnos, docentes, investigadores, etc.) se enfrentan a los materiales escritos que se generan de continuo en el aula puede ser calificada de interpretación o de uso. En este trabajo, tomaremos la postura de que, a los efectos de modelizar la interacción didáctica que surge en torno a los materiales escritos, tanto alumnos como docentes leen los textos que se generan 
en el aula de una manera equivalente a la interpretación, tal y como la entiende Eco. Este aspecto es el punto central del modelo que desarrollaremos en este artículo, y que vamos a denominar aproximación interpretativa (ApIn). El modelo ApIn, por lo tanto, representa una situación «ideal» de aula, en la que todos los agentes didácticos se comportan como «intérpretes» en el sentido de Eco. Hay que insistir en que no es que sostengamos que en el aula los textos sean, de manera natural, interpretados, sino que adoptamos esta visión como un modelo, como una aproximación.

Los puntos fundamentales de nuestro modelo son:

1) Como ya hemos dicho, la base del modelo es considerar que todos los textos generados en el aula son interpretados, en el sentido que da Umberto Eco a este término. En definitiva, tomamos que la interpretación de un texto es una hipótesis sobre la intentio operis del mismo, a partir de la cual se genera un lector modelo y un autor modelo.

2) Asumimos, por lo tanto, que es posible una objetividad en la interpretación de los textos: diferentes intérpretes pueden ponerse de acuerdo sobre una interpretación dada. Este requisito es fundamental para asegurar la falsabilidad de las hipótesis resultantes de la interpretación.

3) Consideraremos que, cuando hablamos de textos de los alumnos, estamos tratando un sistema en el que, aparte de estos dos actores, tenemos casi siempre también dos textos: la «pregunta» que se presenta al alumno y el texto que éste da como respuesta ${ }^{1}$.

4) El modelo ApIn se centra en el papel de los textos como eje comunicativo de la dialéctica alumno-docente (o alumno-investigador). Por lo tanto, dejamos fuera, al menos explícitamente:

- El resto de los actores que pueden intervenir en el proceso. Como veremos en la sección siguiente, la influencia de éstos es «promediada» dentro del modelo.

- Las influencias externas al aula que inciden en el par docente-alumno. Consideramos, como en el caso anterior, que estas influencias son tomadas en cuenta promediadas dentro de las «competencias» tanto del alumno como del docente.

5) Desde el punto de vista ontológico, dentro del modelo ApIn distinguimos cuatro tipos de entidades en el fenómeno didáctico:

- Los alumnos y docentes.

- Los textos que generan alumnos y docentes. Estos dos primeros tipos (individuos y textos) son los «hechos duros», los observables del proceso didáctico.

- Las «competencias» de alumnos y docentes, que tienen un carácter psicológico. En esta categoría se encuadraría el «pensamiento» («ideas», «concepciones», «modelos mentales», etc.) de alumnos y docentes. No entraremos en la cuestión de la naturaleza de estos entes; a los efectos de este artículo nos basta con subrayar su carácter subjetivo.
- Las diferentes hipótesis interpretativas, que son entes lógicos. Se diferencian de las «competencias» en que tienen un carácter externo al individuo: pueden ser formuladas y, dentro de la visión de Eco, falsadas (en términos lógicos, se les puede asignar un valor de verdad).

Cada uno de estos tipos corresponde a una esfera del ser diferente. Desde luego, todas están relacionadas, pero, insistimos, son ámbitos esencialmente distintos.

6) Las interpretaciones juegan un papel esencial: en función de ellas alumnos y docentes (o investigadores) actúan; son el punto de contacto entre la subjetividad (el ámbito de lo psicológico) y la acción.

7) El modelo ApIn es asimétrico: consideramos que el juego de interpretaciones es desencadenado por un textopregunta que formula el docente. Creemos que ésta es una situación didáctica muy común y, de cualquier modo, en trabajos posteriores ampliaremos nuestro modelo a intercambios comunicativos más complejos.

8) Aun cuando tal vez se podría tomar una definición extendida de «texto», que incluyese algunos aspectos de la comunicación oral, limitaremos el modelo ApIn a los materiales escritos. De nuevo, en futuros trabajos extenderemos nuestro enfoque a cualquier tipo de acto didáctico ${ }^{2}$.

9) Dentro del modelo ApIn, alumno y docente-investigador entran en el intercambio de textos con unas maneras, intenciones y expectativas que se pueden considerar normales dentro del entorno didáctico concreto que estemos estudiando. Esta noción de normalidad didáctica puede parecer poco satisfactoria, por vaga, desde el punto de vista metodológico y, sin embargo, está implícita en la idea de contrato didáctico, que tan productiva se ha mostrado en la teoría didáctica (Brousseau, 1986). El contrato didáctico, por su carácter implícito, presupone un «código» de conducta mutua en el aula, al que deben someterse tanto alumnos como docentes; un «código», de hecho, lo cual hace plasmar una «norma», unas «convenciones» de comportamiento. Empleamos convención en un sentido explícitamente pragmático: como describen Acero y sus colaboradores (1986, p. 182), comentando las ideas de Lewis (1969), «una convención es una regularidad en el comportamiento de los miembros de una comunidad, grupo o población, que ésta impulsa, o meramente conserva, porque atenerse a dicha regularidad es un medio satisfactorio de coordinar intereses específicos, y generalizados, de los miembros del grupo». En definitiva, dentro del modelo ApIn asumimos que alumnos y docentes se comportan convencionalmente, en el sentido que acabamos de ver.

10) Asumiremos un principio de la ausencia de ruido en el canal de comunicación. En otras palabras, consideraremos a priori que los textos no están afectados por aspectos incontrolados (erratas, errores tipográficos, etc.) que puedan alterar su interpretación.

11) Tanto el alumno como el docente-investigador son comunicadores óptimamente cooperadores. El concepto de cooperación comunicativa óptima es tremendamente útil en el ámbito de la pragmática filosófica (Grice, 1969, 1989), 
y se refiere a que ambos actores pretenden únicamente transmitir al otro sus intenciones y expectativas comunicativas (no pretenden, por ejemplo, engañarse). Aunque este punto está sin duda alguna relacionado con la idea de normalidad que acabamos de ver, no es necesariamente coincidente: podemos concebir situaciones didácticas en las que la normalidad no suponga una cooperación comunicativa ${ }^{3}$.

12) Docente/investigador y alumno pueden estar aislados «espacialmente» (y de hecho, también, temporalmente) el uno del otro. En otras palabras, el modelo ApIn no se limita a la interacción de aula, e incluye posibilidades como, entre otras, cuestionarios en los que el docente (o el investigador) no esta presente, como, por ejemplo, las investigaciones basadas en cuestionarios remitidos por correo.

\section{ANÁLISIS DEL MODELO APIN}

En la figura 1 mostramos un esquema del modelo ApIn. A su vez, en el anexo I mostramos un ejemplo de aplicación de este modelo a una serie de situaciones comunes en el aula de ciencias.

Las flechas entre las diferentes cajas que aparecen en la figura 1, a excepción de las que han sido destacadas en negrilla (que representan la relación obvia «escribe o genera» o bien «es leído por»), indican una relación de implicación entre ellas (que podría leerse como «si A, entonces $\mathrm{B} »)$, entendida muy genéricamente, no tanto en un sentido lógico «duro» como en un sentido epistemológico o, incluso, psicológico (tal sería el caso, por ejemplo, de la relación de implicación entre «alumno -docente/investigador- empírico» y «competencias del alumno -docente/ investigador-»). Así definidas las relaciones entre los componentes del sistema, cuando en una caja correspondiente a una hipótesis sobre el texto incide más de una flecha, se debe interpretar que pueden existir varias conjeturas independientes, generadas por diferentes vías, sobre el aspecto al que corresponde la caja. En estos casos existe la posibilidad de que estas hipótesis no coincidan; nos encontraríamos, entonces, ante un «conflicto» en el sistema didáctico que surge en torno a un texto. Un aspecto fundamental del modelo ApIn es que estos conflictos son «dirimibles»: algunas interpretaciones pueden ser inaceptables, pueden no resistir la falsación. Esto no implica que no puedan darse varias interpretaciones válidas a un texto dado. Una pregunta puede ser interpretada de manera distinta por diferentes alumnos de un mismo grupo (en el caso extremo, cada alumno podría interpretar el texto de manera distinta; o más aún: un alumno podría proponer varias interpretaciones). A su vez, distintos docentes puedan interpretar de manera divergente una respuesta de un alumno. Pero en todos estos casos, dentro del modelo ApIn todas las interpretaciones han de "sostenerse» dentro del texto en su conjunto. Este carácter falsable de las interpretaciones se recoge implícitamente en el hecho de que cada interpretación es generada por al menos dos vías: por un lado, el lector empírico de un texto, partir de sus competencias; y, por otro, por el mismo texto en sí, independientemente del autor. Esto puede sonar tal vez extraño: ¿cómo puede el autor de un texto y el texto mismo construir autores o lectores modelo distintos? Pues bien, hay que tener en cuenta que el texto, una vez escrito, es autónomo de su autor. Éste no puede garantizar su suerte interpretativa, a no ser, claro, que lo acompañe de algún tipo de explicación adicional en cada lectura; esto es, que el texto sea distinto para cada lector concreto, en cuyo caso estaríamos fuera de las condiciones de nuestro análisis.

En la figura 1 hemos destacado (trazándolas en línea discontinua) las relaciones entre las «interpretaciones» y sus correspondientes «lectores modelo». Con esto queremos indicar el carácter «virtual» de estas relaciones: aun cuando el alumno, desde sus competencias, podría teóricamente enfocar la interpretación de la pregunta como la búsqueda de una hipótesis sobre el «lector modelo» que ésta construye, no creemos que este «distanciamiento metatextual» sea una posición común, al menos explícitamente. Y de igual manera, estamos seguros de que pocos docentes/investigadores se plantearán siquiera el análisis de una respuesta desde una perspectiva análoga.

Como ya hemos indicado, el modelo ApIn es asimétrico, puesto que asumimos que es el docente el que inicia la interacción didáctica. Este hecho está reflejado en la figura 1 por una serie de relaciones («flechas») diferentes para la interpretación de la pregunta y de la respuesta, que han sido destacadas mediante líneas punteadas. Con estas relaciones señalamos los «movimientos» interpretativos distintivos de cada uno de los actores en las circunstancias del aula. Como se puede ver, la diferencia fundamental se centra en el papel de la hipótesis textual sobre el «lector modelo» en cada caso. Desde el punto de vista del docente, esta hipótesis es fundamental: en nuestro modelo, es el punto de arranque del proceso comunicativo. El docente, según su «enciclopedia» (sus conocimientos, sus expectativas, sus experiencias anteriores, etc.), formula una conjetura sobre el alumno al que se dirige su pregunta. Esta conjetura puede ser más o menos explícita (incluso inconsciente). El «alumno - lector modelo» es además una de las fuentes para la interpretación de la respuesta, desde el momento que es el patrón, la «aproximación de orden cero» que sigue el docente investigador en su lectura.

Frente a esto, pasando al polo del alumno, el «docente lector modelo» juega un papel totalmente distinto. Esta hipótesis textual resulta de la interpretación, no la precede. Obviamente, esto se debe a que hemos considerado que la comunicación didáctica es iniciada por el docenteinvestigador. A su vez, éste es el origen de la última asimetría en el modelo, el hecho de que en la generación del texto-respuesta asumamos una doble fuente: por un lado la interpretación de la pregunta, y por otro la «enciclopedia» del alumno. Con esto queremos indicar la posibilidad (frecuente) de que las competencias del alumno no le permitan dar respuesta a la pregunta (de hecho, a su interpretación de la pregunta). Un alumno puede comprender perfectamente qué se pretende de él (esto es, puede interpretar correctamente la pregunta) y, a la vez, ser consciente de que no puede cumplir con estas expectativas. El ejemplo más simple de todo esto se da cuando el alumno «no sabe» la respuesta a una pregunta. 
Figura 1

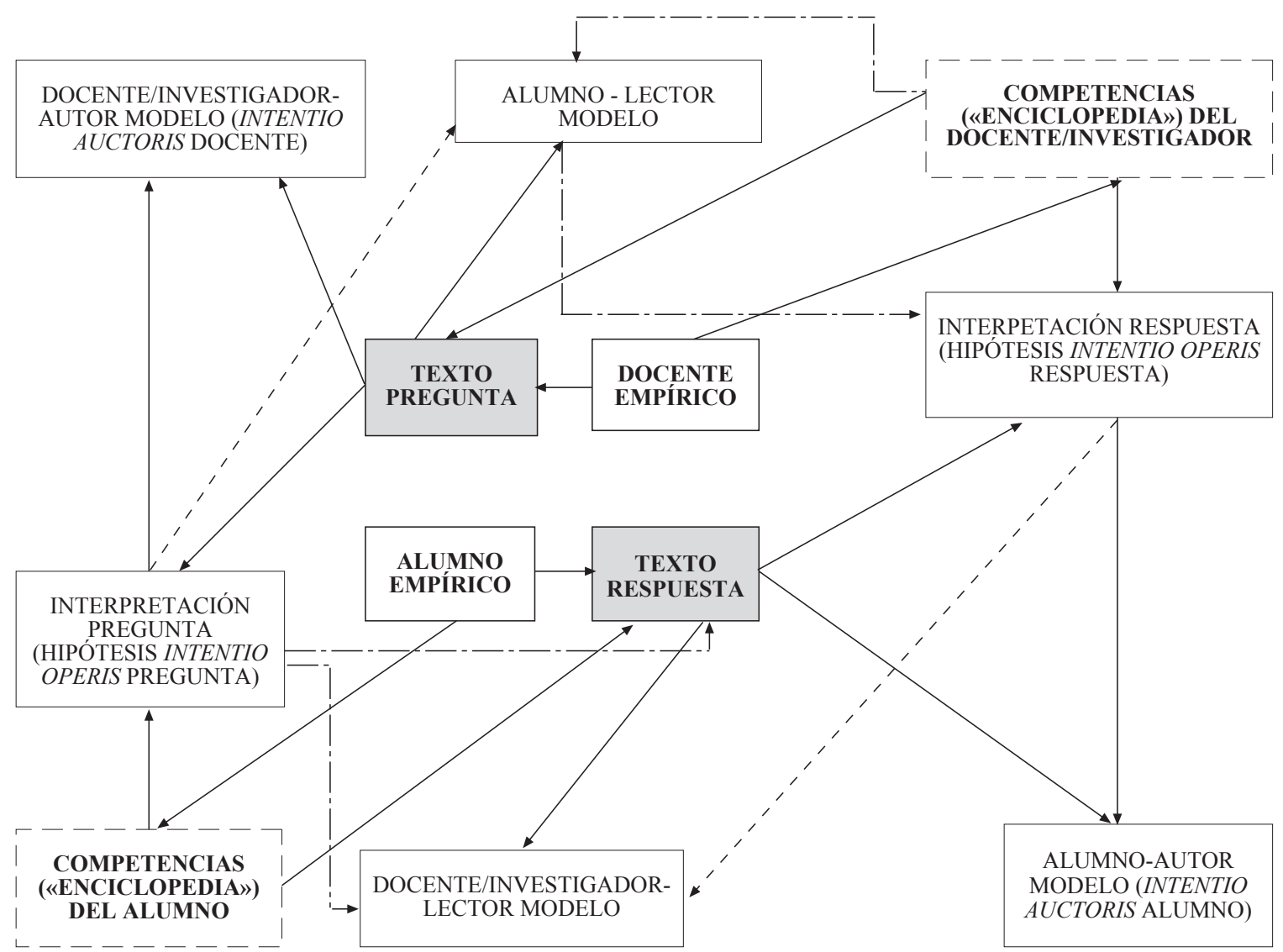

\section{LA EVALUACIÓN DIDÁCTICA EN EL MAR- CO DEL MODELO APIN}

Hemos concluido la sección anterior con una frase deliberadamente provocadora: «El alumno no sabe contestar.» Saber o no saber son los rasgos fundamentales del alumno desde una perspectiva extrema de evaluación. No creemos que haga falta insistir mucho en que, dentro del modelo ApIn, reducir la evaluación a esta dicotomía es ignorar la práctica totalidad del sistema didáctico. La importancia de la evaluación es tan grande que dedicaremos esta sección a su análisis dentro del marco de nuestro modelo.

Ya hemos visto como, dentro de la aproximación ApIn, el modelo de lector que aventura el docente/investigador al formular la pregunta está presente en el acto de interpreta- ción de la respuesta. Este lector modelo es el «patrón», el parámetro primero que guía la lectura de ésta. El docente/ investigador, al formular la pregunta, ha anticipado unas competencias en el destinatario de la misma; estas competencias son las que, al menos en principio, como primera hipótesis, asume en el alumno. Se podría decir que, desde la perspectiva del docente/investigador, y si se quiere como aproximación de orden cero, el «alumno - lector modelo» y el «alumno - autor modelo» deberían coincidir. Como ya dijimos, es una situación análoga, en cierto sentido, con la que se da por parte del alumno al interpretar la pregunta. Sin embargo, existe una diferencia fundamental: el docente/investigador, por su rol de evaluador del proceso docente, no sólo puede sino que debe tener en cuenta explícitamente la posibilidad de el alumno como lector modelo y el alumno como autor modelo no coincidan. La evaluación, 
y el caso particular de la misma que es la calificación, consiste en la confrontación explícita entre estas dos figuras. Dentro de un estilo muy concreto de evaluación, la máxima calificación correspondería al «alumno - autor modelo» que coincidiese completamente con el «alumno - lector modelo» que se postula de partida.

Desde nuestra perspectiva, la evaluación didáctica basada en textos debe ser una metahipótesis sobre este agregado complejo de conjeturas que surge en torno al hecho didáctico. Considerar que la visión del profesor, o del alumno, es la que determina qué ocurre en el aula equivale a una postura reduccionista que dentro del modelo ApIn, por construcción del mismo, no está justificada epistemológicamente $^{4}$. Centrar la evaluación en el docente (esto es, plantearla desde sus expectativas, sus motivaciones, su formación, sus ideas, etc.) o hacerlo en el alumno (asumir que el «pensamiento del alumno» es la única variable que mide el proceso didáctico) son decisiones que tienen una base únicamente ética (o política).

Un último aspecto que debe ser destacado es que, dentro del modelo ApIn, la evaluación global puede ser desglosada en una serie de evaluaciones centradas en aspectos concretos: por ejemplo, la construcción de la hipótesis sobre el alumno como lector modelo por parte del docente. A su vez, la investigación didáctica (que no es sino una evaluación con unos fines que pueden rebasar la dinámica de aula) puede centrarse en las diferentes hipótesis interpretativas como aspectos a investigar. Así, Eco presenta en Lector in fabula (Eco, 1981, pp. 318-320) un caso de investigación experimental orientado a confirmar empíricamente sus ideas sobre el lector modelo, con conclusiones afirmativas. Pues bien, esta búsqueda empírica del lector modelo (o de cualquier otra hipótesis textual) puede ser una estrategia productiva a la hora de guiar la investigación didáctica. De hecho, la investigación sobre el «pensamiento», del alumno o del docente, con todas sus diferentes orientaciones, puede tal vez ser releída desde esta perspectiva. El desarrollo de este último aspecto nos parece de un enorme interés, y es uno de los objetivos que nos marcamos para un desarrollo futuro de nuestras ideas.

\section{SEMÁNTICA Y PRAGMÁTICA EN LA ENSE- NANZA DE LAS CIENCIAS}

Parece obvio asumir a priori que existen unas diferencias fundamentales entre las «enciclopedias» del alumno y del docente/investigador. Desde el punto de vista de los contenidos, estas diferencias derivan del carácter de «experto» del docente, y no son sólo cuantitativas sino también, y eminentemente, cualitativas, e influyen de manera crucial en la interpretación. El alumno, frente a la pregunta, echa mano de unas competencias que están, se podría decir que literalmente, en construcción; su enciclopedia tiene un carácter dinámico, adaptativo. Sin embargo, la enciclopedia del docente, con todas sus connotaciones personales, está basada en gran parte en un saber aceptado y formalizado; empleando la terminología de Chevallard (1991), un «saber sabio» adquirido durante su formación. Si la interpre- tación derivase fundamentalmente de esta «enciclopedia experta», nos encontraríamos con una «lectura desde arriba»; la respuesta es interpretada desde un código rígido, respaldado por un corpus de conocimiento disciplinar que en muchos aspectos es extraño a la cultura y, por lo tanto, al lenguaje del aula. No creemos que haga falta destacar que este hecho puede dar lugar a una incomunicación profunda entre el alumno y el docente/ investigador.

Es en este punto del modelo donde confluyen (o tal vez donde chocan) el lenguaje científico y el lenguaje didáctico. La coexistencia entre estas dos visiones del lenguaje constituye tal vez el principal problema de la didáctica de las ciencias. Un modelo teórico que pretenda describir adecuadamente lo distintivo de la enseñanza de las ciencias debe tener en cuenta esta doble orientación lingüística. En este sentido, el rasgo lingüístico fundamental del método ApIn es que el discurso formalizado, artificial, del saber sabio, y el discurso natural, del saber enseñado, son contemplados simultáneamente.

La dicotomía lenguaje formal - lenguaje ordinario en la enseñanza de las ciencias puede ser vista como una expresión de un problema mucho más general. Dentro de los estudios de filosofía del lenguaje se suelen distinguir dos líneas (o tal vez incluso, dos programas) de investigación bien diferenciadas (Acero et al., 1985, pp. 23-26): por un lado, el estudio de los lenguajes desde una perspectiva lógica (tal sería la orientación de autores como Frege, Russell, el primer Wittgenstein, Carnap o Quine); por otro, el estudio del lenguaje ordinario, del hecho lingüístico como una forma de acción (Austin, el segundo Wittgenstein, Grice o Searle). El primer enfoque se centra en el frente semántico, en el análisis de las relaciones entre lenguaje y realidad (en especial en el problema de la ver$d a d$ ) y en el desarrollo de lenguajes no ambiguos. El lenguaje de la ciencia, al menos idealmente, y por mucho que destaquemos sus aspectos más subjetivos o «literarios» (Locke, 1992), se debe encuadrar en esta corriente. Por otro lado, la segunda corriente es la que antes calificábamos de pragmática. Esta línea trata de poner en claro las relaciones entre el lenguaje y la acción humana. La función «representativa» del lenguaje, su capacidad de hacer asertos sobre el mundo, es tan sólo una de las muchísimas (infinitas, según el Wittgenstein de las investigaciones filosóficas) funciones que el lenguaje toma en el contexto de la vida humana. Empleamos el lenguaje para pedir, para ordenar, para convencer, para engañar, para felicitar... No creemos que haya que argumentar mucho para mostrar que esta dimensión es de una importancia fundamental en el estudio de la interacción didáctica. Y, sin embargo, en el frente semántico, el lenguaje formalizado de la ciencia es también ineludible, aunque sea para ignorarlo o para transformarlo (tal es la tesis de Chevallard).

Sólo en el diálogo entre estas dos tendencias puede surgir una didáctica de las ciencias bien entendida. Limitarse al aspecto semántico nos retrotraería a una enseñanza formalista y rígida, alejada de la subjetividad del alumno, ignorante de la complejidad del aula de ciencias. Pero olvidar el lado semántico supone destruir desde su origen la mis- 
ma idea de enseñanza de las ciencias. Los contenidos científicos, incluyendo el componente formalista de su lenguaje, son irrenunciables, aunque sólo sea por la perogrullada de que, si los ignoramos, no tenemos enseñanza de las ciencias. Otra cosa es que el docente deba tener la libertad (y, por lo tanto, la capacidad: por definición no se es libre para hacer algo para lo que no se está preparado) de tratarlos de la manera que le parezca más adecuada.

\section{CONCLUSIONES}

En este artículo hemos presentado un modelo de la interacción didáctica que tiene lugar en torno al proceso de generación e interpretación de textos, basado en las ideas de la semiótica textual, en especial en los trabajos de Eco. Este modelo, al que hemos denominado aproximación interpretativa (ApIn), analiza una situación didáctica muy concreta, aunque relevante: el caso en el que docente y alumno intercambian textos escritos, en forma de preguntas y respuestas. El punto fundamental del modelo ApIn es la asunción de que estos textos son interpretados, en el sentido que Eco da a este término: la lectura de los textos da lugar a una hipótesis falsable sobre la intentio operis, acerca del sentido del texto por sí mismo, independientemente de su autor.

Hemos mostrado cómo el modelo ApIn aporta una visión de la interacción didáctica que permite racionalizar muchos aspectos de la cultura de aula. En concreto, permite un nuevo enfoque de la evaluación en términos de interpretaciones de los diferentes textos. A su vez, dentro del modelo ApIn encajan de manera natural las vertientes semántica y pragmática de la enseñanza de las ciencias: qué

\section{AGRADECIMIENTOS}

Queremos agradecer, a los profesores Juan Gabriel Morcillo, Manuela Martín Sánchez y Fernando Martín García, su apoyo, así como sus valiosas sugerencias, en la elaboración de este artículo. Este trabajo ha sido financiado parcialmente por la Dirección General de Investigación, del Ministerio de Ciencia y Tecnología, por medio del proyecto de investigación de referencia BFM2000-0033. ciencia se enseña; y cómo se enseña; el debate de los contenidos y el de los métodos.

Como pasa con todos los modelos teóricos, la validez de la aproximación ApIn es tan sólo evaluable a posteriori, en función de los resultados a los que da lugar. En este artículo hemos presentado un primer estudio, muy preliminar, en este sentido. Obviamente, la puesta a prueba de este modelo supone un proyecto de investigación completo y, desde este punto de vista, los objetivos que nos marcamos como continuación de este trabajo son:

- Investigar en mayor profundidad la relación de nuestro modelo pragmático con otras líneas de investigación en didáctica de las ciencias, en especial con las distintas orientaciones de la investigación en las ideas de los alumnos, así como en el pensamiento del profesor.

- En parte como requisito previo al punto anterior, nos proponemos estudiar la estructura de las «enciclopedias», tanto del alumno como del docente investigador, en cuanto a que representan el ámbito psicológico en nuestro modelo.

- A su vez, en el plano metodológico, estamos desarrollando métodos matemáticos semicuantitativos para el análisis de contenido textual, adaptados a nuestro modelo interpretativo (Sánchez Gómez et al., 2001, 2002).

- Nos planteamos poner en práctica un método de evaluación que derive de manera directa del modelo ApIn.

- A medio plazo, nuestro objetivo es extender el enfoque pragmático a todo tipo de materiales comunicativos que se empleen en el aula de ciencias, escritos o no.

\section{NOTAS}

${ }^{1}$ Estamos introduciendo, de hecho, una restricción dentro del modelo, al limitar el texto producido por el docente a un formato interrogativo. A su vez, se asume implícitamente que el texto respuesta del alumno tiene un carácter descriptivo. Un planteamiento más general se podría centrar tal vez empleando la nomenclatura clásica de Austin (1962), en la fuerza ilocucionaria del texto del docente, y en los componentes perlocucionarios del texto respuesta. En este artículo, por simplicidad no entraremos a estudiar este enfoque. 
${ }^{2}$ Aun admitiendo la existencia de diferencias sustanciales entre una respuesta escrita y una oral (Rey-Debove, 1988), en muchos casos tal vez sea posible seguir estudiando esta última como una secuencia lineal de significantes léxicos comparable a un texto. La diferencia fundamental residiría en el código-enciclopedia al que tendríamos que remitirnos en cada caso (Achard, 1988). Sin embargo, una entrevista, por su carácter interactivo, nos parece una situación difícilmente reducible al modelo de texto que nosotros estudiamos.

${ }^{3}$ Por ejemplo, un alumno que copia en un examen está vulnerando el principio de óptima cooperación comunicativa; y, en un examen típico, lo normal es que alguien copie.
${ }^{4}$ Hay que subrayar que, como aproximación, nosotros hemos primado implícitamente el binomio alumno-docente frente al resto de los actores didácticos (la familia del alumno; el resto de los docentes y la dirección del centro escolar; los colectivos profesionales, o sindicales, o políticos en los que se puede encuadrar el docente; los científicos; las autoridades académicas; etc.) y sin duda esto supone una reducción del hecho educativo. Desde nuestra perspectiva, sin embargo, hay una diferencia fundamental entre simplificar la interacción alumno-docente (el núcleo de la interacción didáctica) y «promediar» (a través de las «competencias») la influencia de los aspectos que la rodean. Lo primero suprime de raíz el fenómeno a estudiar y lo segundo (en nuestra opinión), no. Dentro del modelo ApIn, la diferencia entre los roles de alumno y docente, y los del resto de los actores es, por lo tanto, de estatuto epistemológico.

\section{REFERENCIAS BIBLIOGRÁFICAS}

ACERO, J.J., BUSTOS, E. y QUESADA, D. (1985). Introducción a la filosofía del lenguaje. Madrid: Cátedra.

ACHARD, P. (1988). ¿La especificidad de lo escrito es de orden lingüístico o discursivo?, en Catach, N. (comp.) (1996). Hacia una teoría de la lengua escrita, pp. 83-96. Barcelona: Gedisa.

ACHARD, P. (1984). Pour une théorie de la langue écrite. París: Editions du CNRS. Trad. cast. APPLEBEE, A.N. (1984). Writing and Reasoning. Review of Educational Research, 54, pp. 577-596.

AUSTIN, J.L. (1962). How to Do Things with Words (1a. ed.), Oxford: Clarendon Press (2a. ed. rústica, 1974). Oxford: Oxford University Press.

BROUSSEAU, G. (1986). Fondements et méthodes de la didactique des mathématiques. Grenoble: La Pensée Sauvage.

BRUNER, J. (1990). Acts of Meaning. Cambridge, Mass.: Harvard University Press.

COBERN, W.W. (1993). Contextual Constructivism: The Impact of Culture in the Learning and Teaching of Sience, en Tobin, $\mathrm{K}$. (ed.). The Practice of Constructivism in Science Education, pp. 51-69. Hillsdale: Lawrence Erlbaum Associates.

CHEVALLARD, Y. (1991). La transposition didactique. Du savoir savant au savoir enseigné. Grenoble: La Pensée Sauvage.

ECO, U. (1977). Tratado de semiótica general. Barcelona: Lumen.

ECO, U. (1981). Lector in Fabula. Barcelona: Lumen.
ECO, U. (1998). Los límites de la interpretación. Barcelona: Lumen.

GALAGOVSKY, L. (1993). Redes conceptuales: base teórica e implicaciones para el proceso de enseñanza-aprendizaje. Enseñanza de las Ciencias, 11, pp. 301-307.

GALAGOVSKY, L. (1996). Redes conceptuales. Aprendizaje, comunicación y memoria. Buenos Aires: Lugar Editorial.

GALAGOVSKY, L. y CILIBERTI, N. (1994). Redes conceptuales: aplicación a un tema de física de nivel medio. Enseñanza de las Ciencias, 12(3), pp. 338-349.

GIL, D. (1996). New trends in science education. International Journal of Science Edication, 18(8), pp. 889-901.

GODINO, J.D. y BATANERO, C. (1994). Significado institucional y personal de los objetos matemáticos. Recherches en Didactique des Mathématiques, 14(3), pp. 325-355.

GODINO, J.D. y BATANERO, C. (1997). A Semiotic and Anthropological Approach to research in Mathematics Education. Philosophy of Mathematics Education Journal, 10, pp. 1-9.

GRICE, H.P. (1969). Utterer's Meaning and Intentions. Philosophical Review, 78, pp. 147-177. Trad. cast. (1990) (1a. ed.). Las intenciones y el significado del hablante, en Valdés Villanueva, L.M. (ed.). La búsqueda del significado, pp. 481510. Madrid: Tecnos.

GROISMAN, A., SHAPIRO, B. y WILLINSKY, J. (1991). The potential of semiotics to inform understanding of events in 
science education. International Journal of Science Education, 13(3), pp. 217-226.

HALLIDAY, M.A.K. (1978). Language as social semiotic: the social interpretation of language and meaning. Londres: Edward Arnold.

HALLIDAY, M.A.K. y MARTÍN, J.R. (1993). Writing Science. Literacy and Discursive Power. Londres: Falmer Press.

IZQUIERDO, M. y SANMARTÍ, N. (2000). Enseñar a leer y escribir textos de ciencias de la naturaleza, en Jorba, J., Gómez, I. y Prat A. (eds.). Hablar y escribir para aprender. Madrid: Síntesis.

JORBA, J., GÓMEZ, I. y PRAT, A. (eds.) (1998). Parlar i escriure per aprendre. Bellaterra (Barcelona): ICE-UAB. Trad. cast (2000). Hablar y escribir para aprender. Madrid: Síntesis.

KRUGLY-SMOLSKA, E. (1995). Cultural influences in science education. International Journal of Science Education, 17(1), pp. 45-58.

LEMCKE, J.L. (1995). Textual Politics. Londres: Taylor \& Francis.

LEWIS, D. (1969). Convention: A Philosophical Study. Cambridge, Mass.: Harvard University Press.

LOCKE, D. (1992). Science as a Writing. New Haven: Yale University Press.

LYNCH, P.P y JONES, B.L. (1995). Students' alternative frameworks: towards a linguistic and cultural interpretation. International Journal of Science Education, 17(1), pp. 107118.

NOVAK, J.D. (1990). Concept Mapping: A useful device for science education. Journal of Research in Science Teaching, 27(10), pp. 937-949.

NOVAK, J.D. y GOWIN, D.B. (1984). Learning How to Learn. Cambridge: Cambridge University Press. Trad. cast. (1984). Aprendiendo a aprender. Barcelona: Martínez Roca.

PANKRATIUS, W.J. (1990). Building an Organized Knowledge Base: Concept Mapping and Achievement in Secondary School Physics. Journal of Research in Science Teaching, 27(2), pp. 315-333.

PINTÓ, R., ALIBERAS, J. y GÓMEZ, R. (1996). Tres enfoques de la investigación sobre concepciones alternativas. Enseñanza de las Ciencias, 14(2), pp. 221-232.

PRIOR, L. (1997). En Qualitative Research. Theory, Method and Practice. Silverman, D. (ed.), pp. 63-79. Londres: SAGE.

REY-DEBOVE, J. (1988). En busca de la distinción oral/escrito, en Catach, N. (comp.) (1996). Hacia una teoría de la lengua escrita, pp. 97-115. Barcelona: Gedisa.
RIVARD, L.P. (1994). A Review of Writing to Learn in Science: Implications for Practice and Research. Journal of Research in Science Teaching, 31(9), pp. 969-983.

ROWELL, P.M. (1997). Learning in School Science: the Promises and Practices of Writing. Studies in Science Education, 30, pp. 19-56.

RUSSELL, D.R. (1991). Writing in the Academic Disciplines, 1870-1990. Carbondale y Edwardsville: Southern Illinois University Press.

SANMARTÍ, N., IZQUIERDO, M. y GARCÍA, P. (1999). Hablar y escribir. Una condición necesaria para aprender ciencias. Cuadernos de Pedagogía, 281, pp. 54-58.

SCOTT, P. (1998). Teacher Talk and Meaning Making in Science Classrooms: a Vygotskian Analysis and Review. Studies in Science Education, 32, pp. 45-80.

SÁNCHEZ GÓMEZ, P.J., CERVELLÓ, J. y MARTÍN, M. (2001). Una síntesis entre los enfoques cuantitativo y cualitativo en el estudio de materiales escritos: la codificación en red, en Retos de la enseñanza de las ciencias en el siglo XXI. Actas del VI Congreso Internacional sobre Investigación en la Didáctica de las Ciencias, pp. 235236. Barcelona.

SÁNCHEZ GÓMEZ, P.J., MORCILLO, J.G., MARTÍN, M. y SILVÁN, E. (2002). Análisis estructural del contenido de textos de alumnos universitarios sobre estructura atómica, en Relación Secundaria Universidad. Actas de los XX Encuentros de Didáctica de las Ciencias Experimentales, pp. 455-463. La Laguna.

SOLOMON, J. (1994). The Rise and Fall of Constructivism. Studies in Science Education, 23, pp. 1-19.

SPERLING, M. (1996). Revisiting the writing-speaking connection: Challenges for research on writing and writing instruction. Review of Educational Research, 66(1), pp. 53-86.

SUTTON, C.R. (1992). Words, Science and Learning. Buckingham: Open University Press.

SUTTON, C.R. (1996a). Beliefs about Science and Beliefs about Language. International Journal of Science Education, 18(1), pp. 1-18.

SUTTON, C.R. (1996b). The Scientific Model as a Form of Speech, en Welford, G., Osborne, J., Scott, P. (eds.). Research in Science Education in Europe, pp. 143-152. Londres: Falmer Press.

WEININGER, S.J. (1998). Contemplating the Finger. Visuality and the Semiotics of Chemistry. HYLE-An International Journal for the Philosphy of Chemistry, 4(1), pp. 3-27. 
ANEXO I

\section{Un ejemplo de aplicación del modelo ApIn: El modelo ApIn como «racionalizador» de la interacción didáctica}

Veamos una serie de situaciones didácticas típicas:

A. Un docente escribe una pregunta para evaluar el aprendizaje de un determinado aspecto de ciencias. Al leerla, un compañero de centro considera que «tiene un nivel demasiado elevado (o demasiado bajo, o adecuado...) para el grupo».

B. Un alumno, a la salida de la prueba, comenta:

1) «La pregunta era muy fácil (difícil).»

2) «Nunca se sabe lo que quiere este hombre.»

3) «Me he detenido más en esa parte, que es la que le gusta a este tío.»

C. Ante esta pregunta (o ante cualquier otra), el docente se encuentra con que, de manera general, los alumnos del grupo:

1) Contestan todos de manera análoga, en el sentido esperado por el docente.

2) Contestan todos de manera análoga, pero en un sentido diferente al esperado por el docente.

3) Contestan cada uno de una manera.

4) Algunos alumnos (tal vez la mayoría o todos) contestan de manera incoherente o, incluso, disparatadamente.

5) No contestan.

D. Ante la respuesta de un alumno concreto, el docente dice:

1) « Ya sabía yo que esta chica no me iba a fallar!»

2) «Nunca pensé que este alumno no se enterase de nada.»

3) « ¿ómo no se dan cuenta de que no les preguntaba esto!»

4) «Esta respuesta merecería un cero (o un diez).»

Pues bien, todos estos ejemplos se integran cabalmente dentro del modelo ApIn. El docente formula una hipótesis sobre un «alumno lector modelo», que puede o no coincidir con lo que el texto en sí sostiene (caso A). Hay que tener en cuenta que en la gran mayoría de los casos esta conjetura tiene un carácter de idealización estadística: el docente se enfrenta a un aula con una diversidad de alumnos, el «alumno - lector modelo» es en este caso una hipótesis sobre la media del grupo. A la hipótesis textual le superponemos una hipótesis estadística.

El alumno interpreta la pregunta, a partir de sus competencias enciclopédicas (B.1, la valoración «fácil» o «difícil», es en definitiva un juicio sobre la adecuación de la propia enciclopedia enfrentada a un material escrito determinado) y formula una hipótesis sobre el «docente - autor modelo», y de ahí sobre el docente como lector (a veces de una manera casi, diríamos, explícita: B.3). El docente interpreta las respuestas, generando un «alumno - autor modelo», que puede coincidir (C.1) con el «alumno - lector modelo» del que se partía (hipótesis que, obviamente, también puede no ser válida). De manera similar a como veíamos en el párrafo anterior, además de formular hipótesis sobre cada alumno en particular como autor, el docente/investigador puede abstraer de las respuestas un «autor modelo medio». De hecho, en este caso, la homogeneidad de las respuestas apoyaría esta posibilidad. Como se ve, en todo el proceso de lectura/interpretación está presente una tensión entre lo individual y lo grupal, entre el caso y la muestra.

Otra eventualidad posible puede ser que, de las preguntas, «surja» un alumno - autor modelo «medio» suficientemente homogéneo, pero distinto del lector que planteábamos (C.2). Y puede también suceder que las respuestas muestren tal dispersión que no sea posible formular conjetura estadística alguna sobre el alumno como autor (C.3). Por último, una posibilidad límite contemplada en el modelo es que uno de los dos agentes sea incapaz de proponer una interpretación coherente sobre el texto que se le pregunta. Este podría ser el caso de C.4: el docente se ve incapaz de dar sentido a ciertas respuestas en base a su hipótesis inicial sobre el «alumno - lector modelo». De nuevo aparece la tensión entre lo cualitativo y lo cuantitativo: la situación es radicalmente diferente si es tan sólo un alumno el que contesta de esta manera, que si lo hace un número significativo; y en este último caso tampoco es igual que cada contestación incoherente sea característica de un solo alumno, que cuando una de estas respuestas inesperadas es compartida por un número elevado de éstos. Ante estas situaciones hay que plantearse en qué punto empiezan a divergir las interpretaciones de alumnos y docentes/investigadores. En resumen, en C.4 la interpretación por parte del docente queda bloqueada

Esta situación de hipótesis $\angle$ se puede dar también en el polo del alumno como intérprete. Puede ocurrir que éste, por la razón que sea, no sea capaz de construir una interpretación de la pregunta y, por lo tanto, no llegue a postular un docente modelo para la situación concreta. Éste es, desde luego, el caso de B.2, pero tal vez explique también, al menos en algunos casos, la situación C.4. En cuanto a C. 5 , ¿qué puede significar que un alumno no conteste nada? Puede deberse a una imposibilidad de interpretar la pregunta, pero también a que haya preferido no contestar «para no meter la pata». En cualquier caso, cualquier conjetura es ociosa, puesto que, en este caso, no es posible el contraste entre el «alumno - lector modelo» y el «alumno - autor modelo». En rigor, hemos llegado a los límites de la aproximación ApIn: el alumno rehúsa cooperar en la comunicación textual. Desde luego, tenemos un hecho comunicativo, pero éste rebasa el formato escrito, y se requiere un modelo pragmático más general. En artículos venideros nos centraremos en este tema. 